\title{
CHAPTER 11
}

\section{THERMO-CHEMICAL STRUCTURE OF THE LOWER MANTLE: SEISMOLOGICAL EVIDENCE AND CONSEQUENCES FOR GEODYNAMICS}

\author{
FRÉDÉRIC DESCHAMPS ${ }^{1,3}$, JEANNOT TRAMPERT $^{1}$, AND \\ PAUL J. TACKLEY ${ }^{2}$
}

\author{
${ }^{1}$ Department of Earth Sciences, Utrecht University, Budapestlaan 4, PO Box 80021, 3508 TA Utrecht, \\ The Netherlands; \\ E-mail: frederic.deschamps@erdw.ethz.ch \\ ${ }^{2}$ Department of Earth and Space Sciences, University of California, 595 Charles Young Drive East, Los \\ Angeles, California 90095, USA \\ ${ }^{3}$ Now at Institute of Geophysics, Swiss Federal Institute of Technology, HPP Honggerberg L8.1, 8083 \\ Zurich, Switzerland
}

\begin{abstract}
We combine recent progress in seismic tomography and numerical modeling of thermochemical convection to infer robust features on mantle structure and dynamics. First, we separate the observed density anomalies into their thermal and compositional contributions. The tomographic maps of thermo-chemical variations were computed using a new approach that combines a careful equation of state modeling of the lower mantle, independent constraints on density from probabilistic tomography, and a full statistical treatment for uncertainties analyses. We then test models of thermo-chemical convection against these density components. We compute synthetic anomalies of thermal and compositional density from models of thermo-chemical convection calculated with the anelastic approximation. These synthetic distributions are filtered to make meaningful comparisons with the observed density anomalies. Our comparisons suggest that a stable layer (i.e., that no domes or piles are generated from it) of dense material with buoyancy ratio $B \geq 0.3$ is unlikely to be present at the bottom of the mantle. Models of piles entrained upwards from a dense, but unstable layer with buoyancy ratio $B \sim 0.2$, explain the observation significantly better, but discrepancies remain at the top of the lower mantle. These discrepancies could be linked to the deflection of slabs around $1000 \mathrm{~km}$, or to the phase transformation at $670 \mathrm{~km}$, not included yet in the thermo-chemical calculations.
\end{abstract}




\section{INTRODUCTION}

Inferring the thermo-chemical structure of the mantle is a key problem in geophysics. Both thermal and chemical sources contribute to lateral variations in density, and the mode of mantle convection strongly depends on the relative strength of these two sources. It is the goal of seismology to map and quantify these contributions. Although seismic data represent our most detailed information on Earth structure, their interpretation raises difficulties that are often underestimated (Trampert and van der Hilst, 2005). For instance, the low shear-wave velocity structures in the deep mantle observed beneath Africa and the Pacific in many tomographic models (e.g., Su and Dziewonski, 1997; Vasco and Johnson, 1998; Masters et al., 2000) are usually referred to as "superplumes" and interpreted as hotter than average, and hence buoyant material (Forte and Mitrovica, 2001; Romanowicz and Gung, 2002). This interpretation implicitly assumes that shear-wave velocity anomalies are a reasonable proxy for temperature and density. It has been argued that in the uppermost mantle compositional effects do not strongly influence seismic velocity (Goes et al., 2000). In the lower mantle, however, pressure effects reduce the influence of temperature, and compositional effects may dominate seismic velocity anomalies (Anderson, 2001). Moreover, we should not forget that even if chemical anomalies do not significantly contribute to seismic velocity anomalies, they may still contribute to density anomalies, which are the important parameters in terms of dynamics.

Lateral variations of temperature and composition play a key role in linking geophysical observations and geodynamics. In recent years, mounting evidence suggested that chemical variations are present in the lower mantle (Ishii and Tromp, 1999; Kellogg et al., 1999; Forte and Mitrovica, 2001; Karato and Karki, 2001; Saltzer et al., 2001; Deschamps and Trampert, 2003), but difficulties remain to quantify thermo-chemical variations from seismology, and to relate them to thermo-chemical convection. Unless independent constraints on mass density are available, the distributions of temperature and composition inferred from tomographic models are not robust (Deschamps and Trampert, 2003).

Recent progresses shed new light on the link between seismology and geodynamics. Probabilistic tomography (Resovsky and Trampert, 2003) estimated independent and robust constraints on the lateral variations of density and seismic velocities, which can be converted into thermo-chemical variations (Trampert et al., 2004). Models of thermo-chemical convection that include the anelastic (e.g., Tackley, 1998) or extended Boussinesq (e.g., Schott and Yuen, 2004) approximation predict more realistic density contrasts. In this chapter, we investigate the geodynamical consequences of these improvements.

\section{DENSITY ANOMALIES FROM THERMO-CHEMICAL CONVECTION}

A variety of experimental (Olson and Kincaid, 1991; Davaille, 1999) and numerical (Hansen and Yuen, 1988; Christensen and Hofmann, 1994; Kellogg et al., 1999; Tackley, 1998, 2002; Schott and Yuen, 2004) models of thermo-chemical convection 
have been developed, showing strong stratification or a more complex structure, depending on the fluid properties and on the buoyancy ratio. A major improvement was to perform calculations that account for fluid compressibility, either using the anelastic (Tackley, 1998), or extended Boussinesq (Schott and Yuen, 2004) approximation. In these approximations, some dissipation is present, and thermo-chemical properties are allowed to vary with depth through the prescription of a reference model. The decrease of thermal expansion with depth, which is well documented from mineral physics data (e.g., Anderson, 1995), is particularly important because it weakens the thermal buoyancy in the lowermost mantle. As a result, chemical stratification and the development of thermo-chemical domes require a smaller buoyancy ratio than in the classical Boussinesq approximation. The mode of convection is therefore controlled by several key parameters, including the buoyancy ratio, the thermal expansion, and the initial composition. Each convection regime predicts a distribution of temperature and composition that we want to test against seismological observations. In this section, we show how to compute the density variations due to the variations of temperature and composition predicted by several models of thermo-chemical convection (Tackley, 2002).

Details of the numerical modeling are described in Tackley $(1998,2002)$. The equations of conservation of mass, energy and momentum are solved for an anelastic, compressible fluid with infinite Prandlt number. Calculations are made in a 3-D Cartesian box of dimensions $4 \times 4 \times 1$. The fluid is cooled on the top, and heated both from below and from within. The top and bottom boundaries are isothermal, but some cases with zero heat flow at each point (i.e., no bottom heating) have also been considered. The viscosity depends on depth and composition. The viscosity increases smoothly by a factor of 10 across the fluid layer, and an additional viscosity jump equal to 30 is imposed at $z=660 \mathrm{~km}$. The dense material is less viscous than the light material by a factor of 100. Calculations involve a depth-dependent reference thermodynamic model (Tackley, 1998), including temperature, density, thermal expansion, and thermal conductivity. The chemical field is represented using 10 million tracer particles dispatched everywhere in the box. Two types of particles are considered, one for regular material, and one for dense material. The fraction of dense particles $C$ in each cell varies between $C=0$ when the cell is filled with regular material only, and $C=1$ when the cell is filled with particles of dense material only. The chemical density contrast $\Delta \rho_{c}^{\max }$ between dense and regular material is acting against the thermal buoyancy due to the thermal density contrast across the fluid layer $\Delta \rho_{T}^{\max }=\alpha_{S} \rho_{s} \Delta T_{s}$, where $\alpha_{s}$ and $\rho_{s}$ are the thermal expansion and density at zero pressure, and $\Delta T_{S}$ the superadiabatic temperature difference between the top and the bottom of the layer. The relative strength of chemical and thermal buoyancies is measured by the global buoyancy ratio

$$
B \equiv \frac{\Delta \rho_{c}^{\max }}{\alpha_{s} \rho_{s} \Delta T_{S}},
$$

which is used to define the chemical Rayleigh number. 
The density at temperature $T$ and regular material $(C=0)$ can be computed using the depth-dependent reference state (temperature $T_{\text {ref }}$ and thermal expansion $\alpha_{\text {ref }}$ ),

$$
\rho_{T}=\rho_{\text {ref }}\left[1-\alpha_{\text {ref }}\left(T-T_{\text {ref }}\right)\right] .
$$

The density at temperature $T_{\text {ref }}$ and fraction of dense material $C$ can be expressed as a function of the buoyancy ratio (Eq. 1),

$$
\rho_{c}=\rho_{s}\left[1+B C \alpha_{s} \Delta T\right] .
$$

If $\bar{T}$ and $\bar{C}$ are the average temperature and the average fraction of dense particles at a given depth, the relative thermal and chemical density anomalies are

$$
\begin{aligned}
\mathrm{d} \ln \rho_{T} & =-\frac{\alpha_{\mathrm{ref}}(T-\bar{T})}{\left[1-\alpha_{\mathrm{ref}}\left(\bar{T}-T_{\mathrm{ref}}\right)\right]}, \\
\text { and } \quad \mathrm{d} \ln \rho_{c} & =-\frac{B(C-\bar{C})}{\left(1+B \bar{C} \alpha_{s} \Delta T\right)} \alpha_{s} \Delta T_{s} .
\end{aligned}
$$

We computed synthetic $\mathrm{d} \ln \rho_{T}$ and $\mathrm{d} \ln \rho_{c}$ for four numerical models with buoyancy ratio equal to $0.2,0.3$ or 0.4 and percentage of dense material equal to $10 \%$ or $30 \%$ (Table 1). The bottom boundary is isothermal in all cases, except for $B=0.4$, $X=10 \%$, in which the heat flow is put to zero everywhere on the boundary. Calculations were initialized with a geotherm from similar 2-D calculations that have reached a secular equilibrium. The models we tested are snapshots representative of long-term evolution, and the dimensional evolution times are listed in Table 1. Figure 1 shows isosurfaces of the fraction of dense particles and the non-dimensional temperature, and Figure 2 shows $\bar{T}, \bar{C}$, and the root mean square (RMS) of $\mathrm{d} \ln \rho_{T}$ and $\mathrm{d} \ln \rho_{c}$ as a function of depth. For $B=0.3, X=30 \%$, a thick $(\sim 500 \mathrm{~km})$ stable layer of dense and hot material sediments at the bottom of the fluid (Fig. 1, plots $\mathrm{i}-1$ ). The RMS of $\mathrm{d} \ln \rho_{T}$ and $\mathrm{d} \ln \rho_{c}$ (Fig. 2, green curves) indicate that this layer is thermally and chemically homogeneous, and that most of the lateral variations of temperature and composition are located between 1600 and $2400 \mathrm{~km}$. In the case $B=0.4, X=10 \%$, a stable layer is also present at the bottom of the fluid, but it is much thinner and does not completely cover the bottom boundary (Fig. 1, plots $\mathrm{m}-\mathrm{p}$ ). The Strongest lateral variations of thermal and chemical density are found

Table 1. Four models of thermo-chemical convection ${ }^{\mathrm{a}}$

\begin{tabular}{lccll}
\hline$B$ & $X(\%)$ & $t(\mathrm{~Gy})$ & Bottom boundary condition & Convective pattern \\
\hline 0.2 & 10 & 1.1 & Isothermal & Piles \\
0.3 & 10 & 6.0 & Isothermal & Discontinuous spokes \\
0.3 & 30 & 2.7 & Isothermal & Thick stable layer \\
0.4 & 10 & 4.2 & Zero heat flux at each point & Thin stable layer \\
\hline
\end{tabular}

a $B$ is the buoyancy ratio, $X$ the fraction of dense material and $t$ the evolution time. 


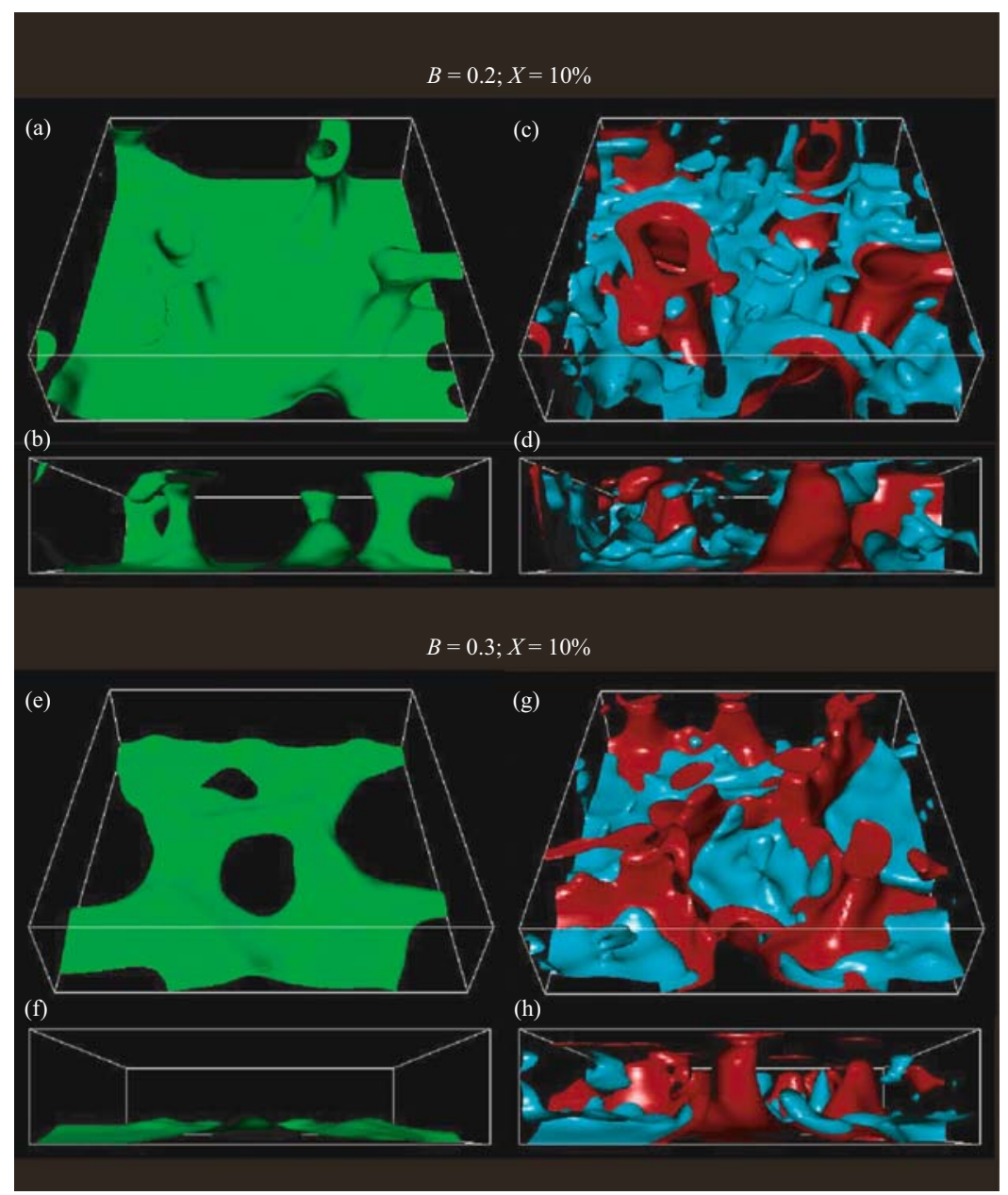

Figure 1. Isosurfaces of non-dimensional temperature (red and blue) and fraction of dense particles (green) for thermo-chemical convection models for various buoyancy ratios $(B)$ and fractions of dense particles $(X)$. Contour levels are $T=0.0375$ (red), $T=-0.0375$ (blue), and $C=0.5$ (green). The cases considered are (a-d) $B=0.2$ and $X=10 \%$; (e-h) $B=0.3$ and $X=10 \%$; (i-1) $B=0.3$ and $X=30 \%$ (green curves); and (m-p) $B=0.4$ and $X=10 \%$.

between 2500 and $2750 \mathrm{~km}$ (Fig. 2, blue curves). For $B=0.3, X=10 \%$, the fluid is organized in a discontinuous spoke pattern of dense and hot material (Fig. 1, plots e-h). Strong lateral variations of density are present from $z=2400 \mathrm{~km}$ down to the bottom of the fluid (Fig. 2, red curves). Note that the two previous cases do not show significant lateral variations of composition for depths shallower than $2000 \mathrm{~km}$. Finally, for $B=0.2, X=10 \%$, piles are generated in the deep mantle from 


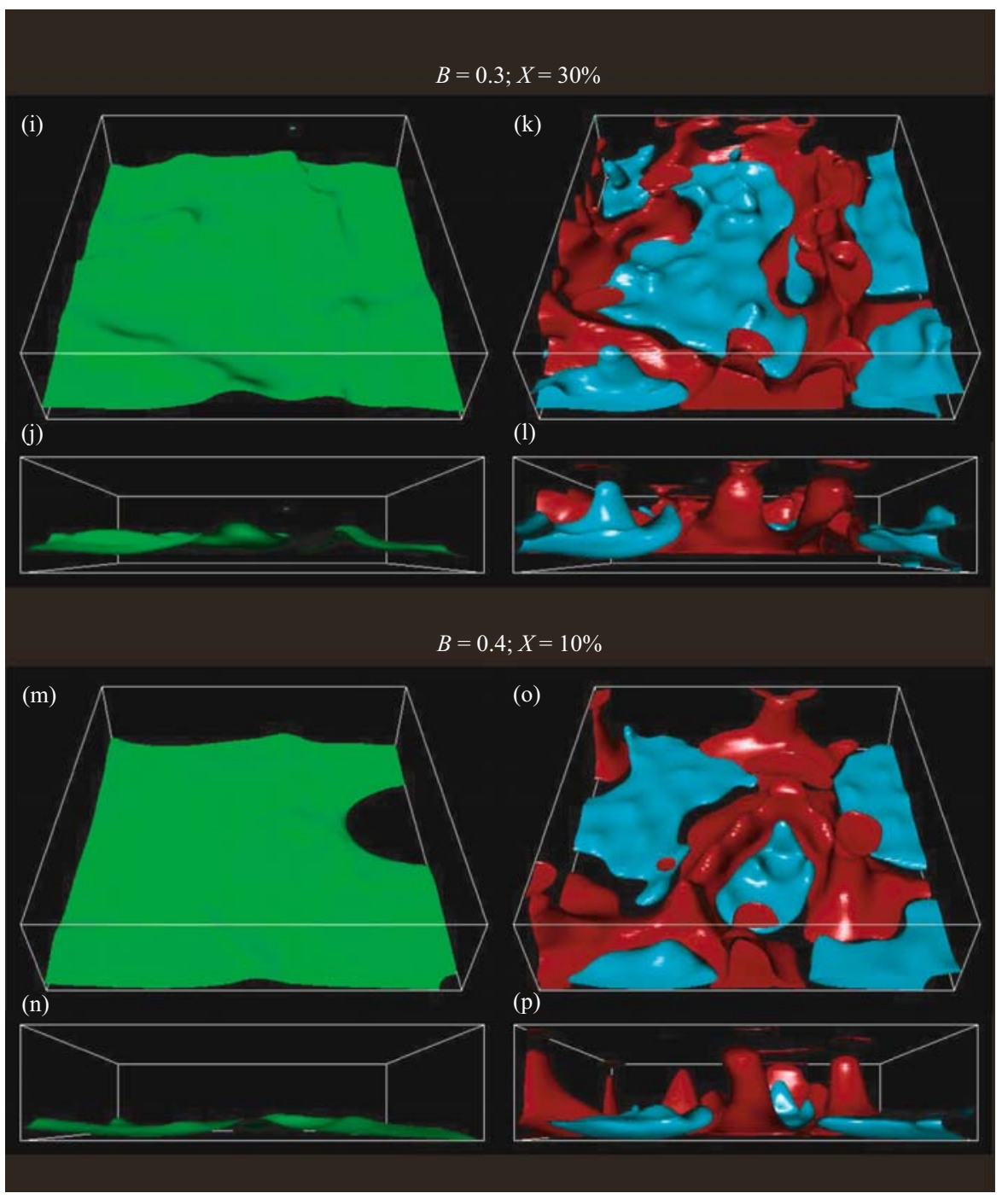

Figure 1. (Continued).

a layer of dense material, and are entrained upwards (Fig. 1, plots a-d). Chemical density anomalies are increasing progressively with depth, and drop dramatically to zero in the last $100 \mathrm{~km}$ (Fig. 2, orange curve). Some significant variations of density (thermal and chemical) are also observed in the upper half layer of the fluid. It is interesting to note that only this case predicts chemical variations throughout the fluid layer. 


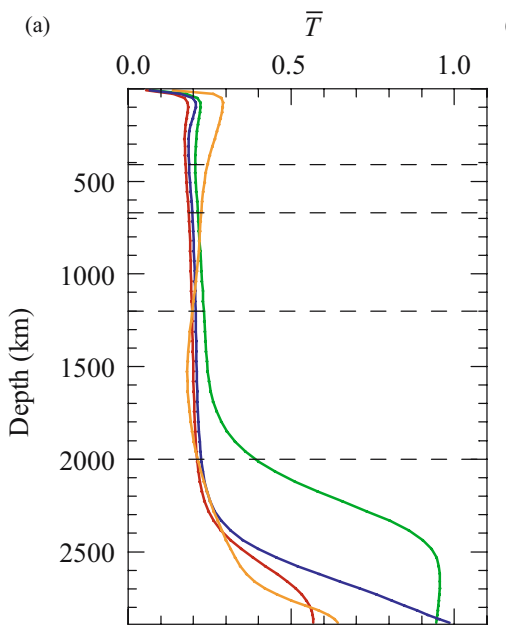

(b) $\quad \operatorname{RMS}\left(\mathrm{d} \ln \rho_{T}\right)(\%)$
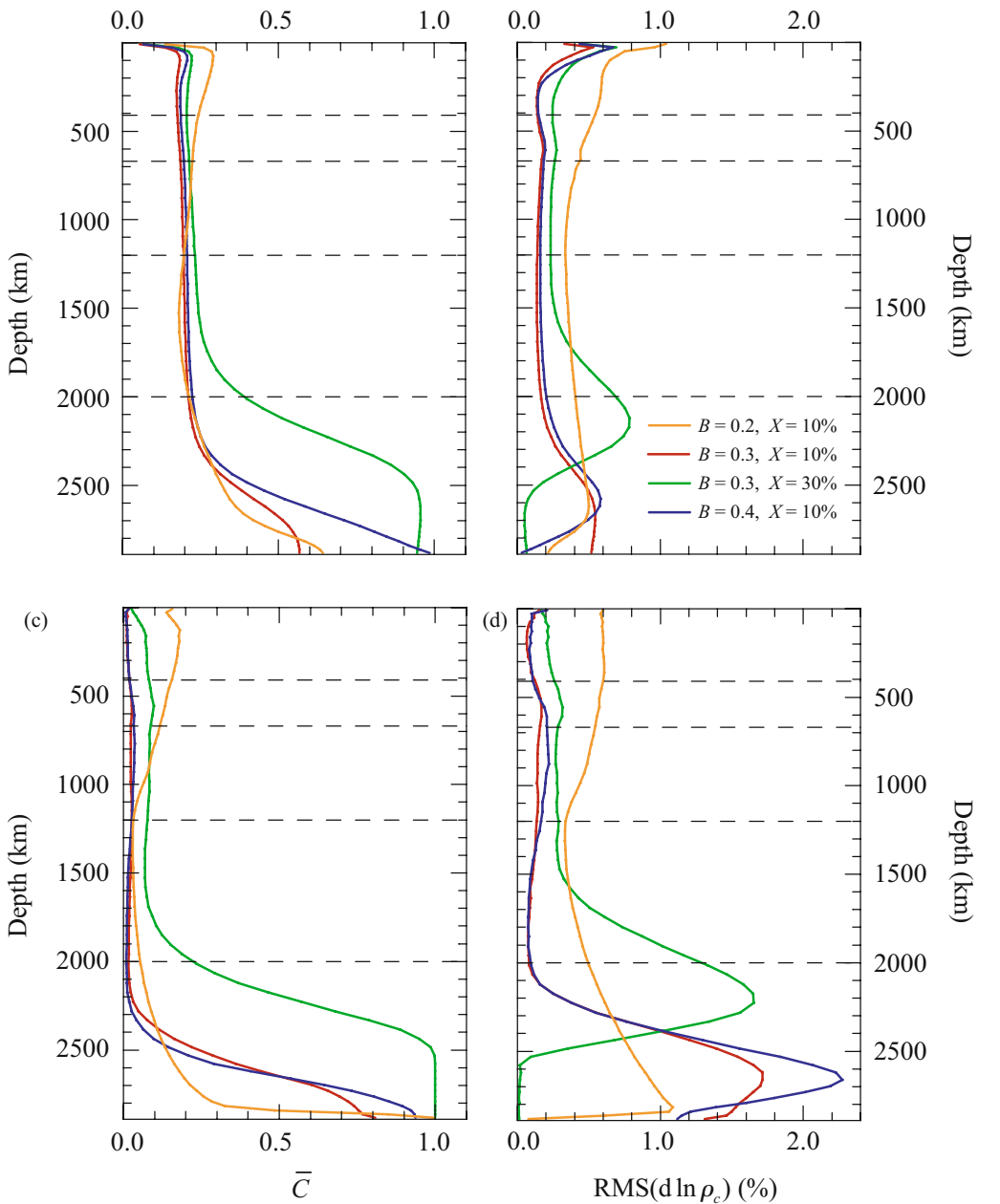

Figure 2. Profiles of (a) average temperature, (b) RMS of lateral variations of thermal density, (c) average fraction of dense particles, and (d) RMS of lateral variations of chemical density, for each model of thermo-chemical convection plotted in Figure 1. Orange curves are for $B=0.2$ and $X=10 \%$, red curves for $B=0.3$ and $X=10 \%$, green curves for $B=0.3$ and $X=30 \%$, and blue curves for $B=0.4$ and $X=10 \%$.

\section{PROBABILISTIC SEISMIC TOMOGRAPHY}

Seismic velocities alone cannot resolve the interconnection between temperature and composition. Independent constraints on density anomalies are needed to infer correct variations of temperature and composition. Early constraints on density from normal 


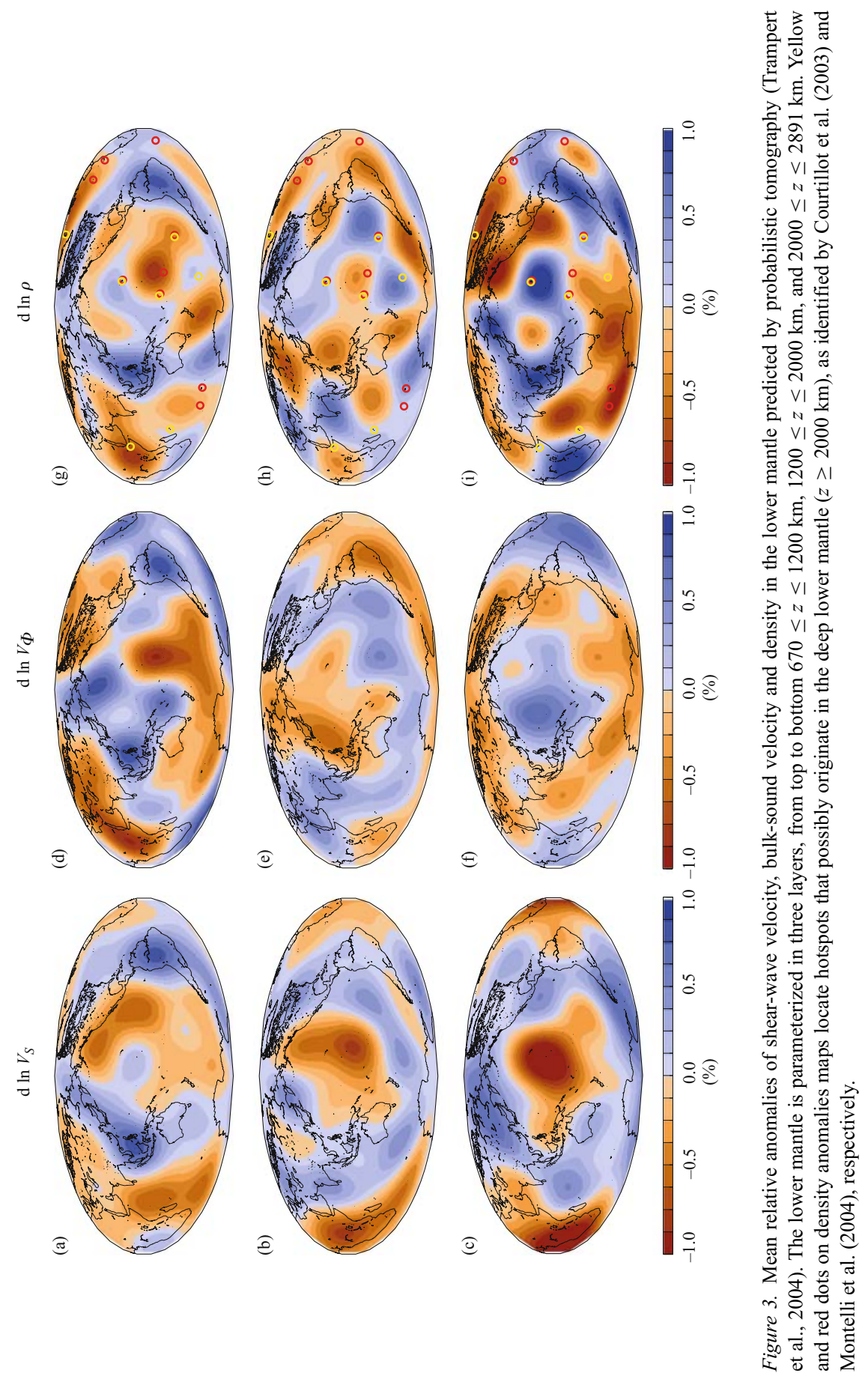


Table 2. Statistics for tomographic observables and thermo-chemical parameters ${ }^{\mathrm{a}}$

\begin{tabular}{llllllllll}
\hline $\begin{array}{l}\text { Layer } \\
(\mathrm{km})\end{array}$ & & $\begin{array}{l}\mathrm{d} \ln V_{S} \\
(\%)\end{array}$ & $\begin{array}{l}\mathrm{d} \ln V_{\Phi} \\
(\%)\end{array}$ & $\begin{array}{l}\mathrm{d} \ln \rho \\
(\%)\end{array}$ & $\begin{array}{l}d T \\
(\mathrm{~K})\end{array}$ & $\begin{array}{l}d X_{p v} \\
(\%)\end{array}$ & $\begin{array}{l}d F e \\
(\%)\end{array}$ & $\begin{array}{l}\mathrm{d} \ln \rho_{T} \\
(\%)\end{array}$ & $\begin{array}{l}\mathrm{d} \ln \rho_{c} \\
(\%)\end{array}$ \\
\hline $670-1200$ & $A$ & 0.77 & 0.87 & 0.79 & 340 & 11.9 & 1.8 & 0.64 & 0.80 \\
& $\operatorname{RMS}(\sigma)$ & 0.16 & 0.34 & 0.26 & 180 & 5.5 & 0.75 & 0.38 & 0.34 \\
$1200-2000$ & $A$ & 0.77 & 0.57 & 0.70 & 311 & 8.3 & 1.2 & 0.41 & 0.45 \\
& $\operatorname{RMS}(\sigma)$ & 0.12 & 0.22 & 0.28 & 112 & 3.0 & 0.55 & 0.15 & 0.22 \\
$2000-2891$ & $A$ & 1.21 & 0.66 & 1.05 & 475 & 9.3 & 2.6 & 0.44 & 0.95 \\
& $\operatorname{RMS}(\sigma)$ & 0.12 & 0.26 & 0.48 & 198 & 3.6 & 0.86 & 0.19 & 0.37 \\
\hline
\end{tabular}

${ }^{\text {a }} A$ is the maximum amplitude of the average model, and $\operatorname{RMS}(\sigma)$ is the root mean square of the uncertainty.

modes were inferred by Ishii and Tromp (1999). More recent studies (Resovsky and Trampert, 2003) have developed the technique of probabilistic seismic tomography, which gives robust likelihoods for long wavelength models (spherical harmonic degree 2, 4, and 6) bulk-sound speed, shear wave speed, density and boundary topography in the mantle, from normal mode splitting and surface wave data. Probabilistic tomography explores the model space using a neighborhood algorithm (Sambridge, 1999a, 1999b) and estimates the likelihood of points in the model space by testing them against observables. The likelihoods of Resovsky and Trampert (2003), hereafter referred to as RT246g, are fully compatible with the observed gravity anomalies, and can thus be seen as a compact representation of the available seismic and gravity data. To a very good approximation, they can be represented as Gaussian distributions, and their standard deviation is remarkably stable within each layer. Figure 3 shows the mean of the distributions for $\mathrm{d} \ln V_{S}, \mathrm{~d} \ln V_{\Phi}$ and $\mathrm{d} \ln \rho$ in each layer, and Table 2 lists the root mean square (RMS) of the uncertainties (defined as one standard deviation around the mean value). In the deepest layer (2000 $\leq z \leq 2891)$, there is a general agreement between the $\mathrm{d} \ln V_{S}, \mathrm{~d} \ln V_{\Phi}$ of RT246g and those predicted by previous models (Su and Dziewonski, 1997; Vasco and Johnson, 1998; Masters et al., 2000). Like previous studies, RT246g shows regions of low shearwave velocity beneath Africa and the Pacific. Assuming that $\mathrm{d} \ln V_{S}$ scales with lateral variations of temperature, these regions are usually interpreted as "superplumes". However, a quick look to the density anomalies of RT246g (Fig. 3i) clearly shows that these features are denser than the surrounding mantle, and hence not buoyant.

\section{LINKING OBSERVABLES TO THERMO-CHEMICAL PARAMETERS}

\subsection{Sensitivities of density and seismic velocities to temperature and composition}

Density and thermo-elastic properties of a lower mantle aggregate depend on temperature, pressure and composition. A careful modeling of these effects is essential to correctly interpret seismological observations. The basic ingredients are the density 
and elastic parameters at ambient temperature and pressure, and a set of relations (the equation of state, EOS) that extrapolate these ambient values at mantle temperature and pressure. To calculate thermo-elastic properties at any depth and temperature, we have built a parameterization that combines EOS modeling, experimental values of thermo-elastic parameters at ambient conditions, and existing ab initio data calculations (Deschamps and Trampert, 2004). The latter are used to check the consistency of high-temperature and high pressure extrapolations. We are thus able to calculate velocities and density of a given mineralogical composition at mantle pressures and temperatures. Several sources of uncertainties, mainly the error bars on the ambient thermo-elastic parameters and the assumed potential temperature and assemblage composition, contribute to uncertainties on the extrapolated thermoelastic parameters. The latter are obtained by varying thermo-elastic parameters within their error bars, and average temperature and composition within reasonable range. PREM is used as an additional constraint to reduce the uncertainty range.

By repeating the calculations of elastic moduli and density at high-temperature and high-pressure for slightly different values of temperature and composition, we estimate the partial derivatives (or sensitivities) of density and seismic velocities to temperature and several compositional parameters as a function of depth. The sensitivities plotted in Figure 4 (used in Trampert et al., 2004) account for the most recent corrections for iron (Kiefer et al., 2002; Kung et al., 2002). Compared to previous work (Trampert et al., 2001; Deschamps and Trampert, 2003), sensitivities of shear-wave velocities to composition are different, mainly due to the iron correction for the pressure derivatives of the moduli of magnesio-wüstite (Kung et al., 2002), not included in previous work.

Sensitivities of both shear and bulk sound velocities have similar properties (sign and variation with depth): sensitivities to temperature are negative throughout the mantle and increase with depth; sensitivities to perovskite are positive and decrease with depth; and sensitivities to iron are negative and decrease with depth. A consequence of this similarity is that seismic velocities alone cannot discriminate between thermal and compositional effects. Sensitivities of density (Fig. 4c) are clearly different. The sensitivity to perovskite is negligible, whereas the sensitivity to iron is positive throughout the mantle and increases with depth. Density therefore carries essential information to separate thermal and compositional effects. Another important feature in Figure $4 \mathrm{c}$ is that the sensitivity of density to temperature (i.e., the thermal expansion of the aggregate) is decreasing in amplitude with depth. As a consequence, for a fixed temperature anomaly, the strength of the thermal buoyancy is also decreasing with depth.

\subsection{Converting density and seismic velocities}

Several thermo-chemical sources contribute to the observed anomalies of density and seismic velocities. Knowing the sensitivities of density and seismic velocities 

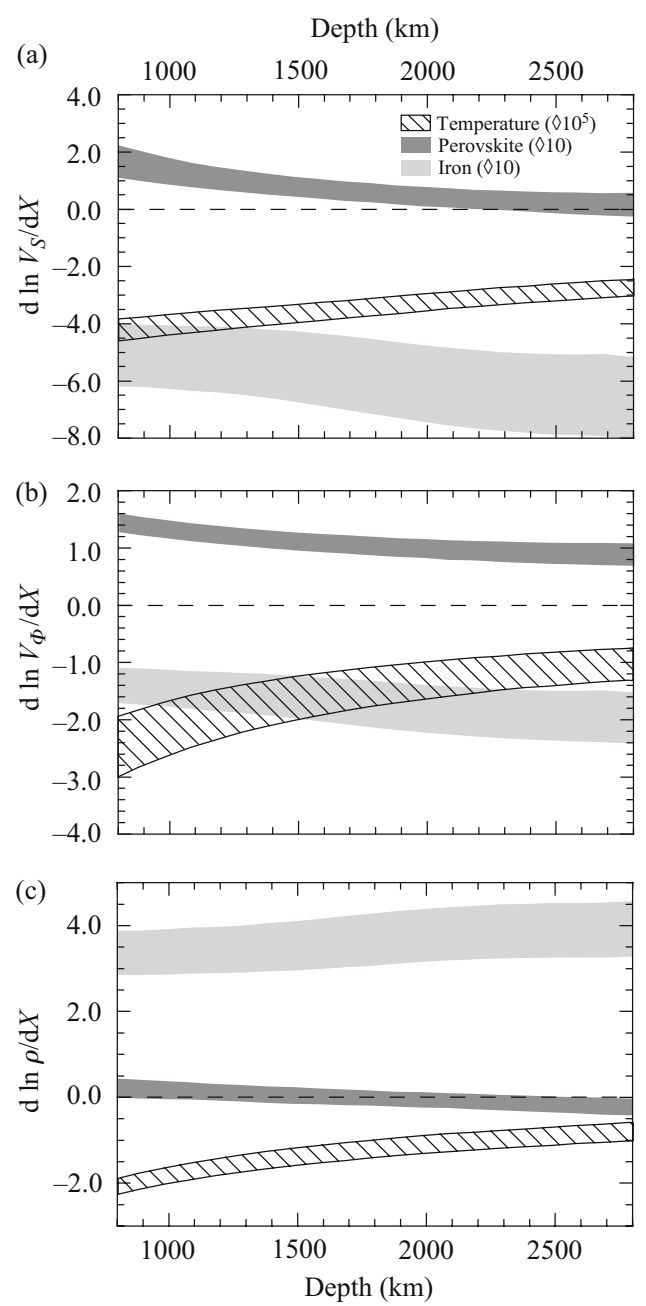

Figure 4. Sensitivities of seismic velocities and density to temperature (dashed areas), perovskite (dark grey areas) and iron content (light grey areas) as a function of depth. Dashed and shaded areas cover one standard deviation around the mean values. Mean and standard deviations are estimated from a collection of possible models that fit PREM within $1 \%$, and are generated by varying thermo-elastic parameters within their error bars, and the average temperature and composition within reasonable ranges. For plotting convenience, sensitivities to temperature are multiplied by $10^{5}$, and sensitivities to perovskite and iron by 10 .

to these sources, one can reconstruct synthetic anomalies by adding possible contributions, and compare them to the observed anomalies. A difficulty is to decide which contributions are significant, and which are not. Lateral variations of temperature $(d T)$ are of course a major source. Partial melt is locally generating dramatic 
effects, but is limited in extension (Williams and Garnero, 1996) and has therefore limited effects on the global tomography (Deschamps and Trampert, 2003). Compositional (or chemical) variations are a significant contribution, but they consist in a variety of sources that must be treated independently. Since the mantle aggregate consists in a silicate phase (perovskite) and magnesio-wüstite, it is natural to consider the variations in silicate. In the remainder of the chapter, we refer to these variations as variations in the volumetric fraction of perovskite $d X_{p v}$. In the lower mantle, iron is incorporated in both perovskite and magnesio-wüstite, and induces strong changes in the properties of these minerals. Changes in the elastic moduli (and their derivatives) are moderate for perovskite (Kiefer et al., 2002), but very strong for magnesio-wüstite (Kung et al., 2002). In addition, the density of both perovskite and magnesio-wüstite strongly increases with the iron fraction. Figure 4 clearly shows that the variations in the global volumetric fraction of iron $\mathrm{dFe}$ strongly contribute to the anomalies of density and seismic velocity. At lower mantle pressure, subducted oceanic crust results in material enriched in calcium compared to average aggregate. If oceanic slabs reach the bottom of the mantle, the fraction of calcium $\left(X_{\mathrm{Ca}}\right)$ may vary laterally. Calcium is incorporated in perovskite, and induces moderate changes in the elastic properties and very small changes in density. Because in our modeling we considered $\mathrm{CaSiO}_{3}$ as a pole of perovskite, the sensitivities shown in Figure 4 implicitly account for variations in calcium. Practically, however, the differences between these sensitivities and those obtained for $X_{\mathrm{Ca}}=0$ are not significant. In addition, the sensitivities of density and seismic velocities to calcium are one order of magnitude smaller than those for iron. For these reasons we neglected the contribution of the variations in calcium to the relative anomalies of density and seismic velocities. The fraction of aluminum in perovskite is also expected to vary if subducted crust reaches the lower mantle. The effects of aluminum on the thermo-elastic properties of perovskite are still debated. Jackson et al. (2004) reported important modification in the shear modulus, but no agreement could be found for the bulk modulus (Daniel et al., 2004; Jackson et al., 2004; Yagi et al., 2004). In addition, there are presently no robust estimates for the pressure and temperature derivatives of the elastic moduli. Because the real effects of aluminum are still uncertain, we did not account for them, but potentially they can have a large influence. At first order, it is therefore reasonable to parameterize compositional variations as a function of the variations in perovskite and iron, and the relative anomalies of shearwave velocity $\left(\mathrm{d} \ln V_{S}\right)$, bulk sound velocity $\left(\mathrm{d} \ln V_{\Phi}\right)$, and density $(\mathrm{d} \ln \rho)$ can be written

$$
\begin{aligned}
\mathrm{d} \ln V_{S} & =\frac{\partial \ln V_{S}}{\partial T} d T+\frac{\partial \ln V_{S}}{\partial X_{p v}} d X_{p v}+\frac{\partial \ln V_{S}}{\partial F e} d F e \\
\mathrm{~d} \ln V_{\Phi} & =\frac{\partial \ln V_{\Phi}}{\partial T} d T+\frac{\partial \ln V_{\Phi}}{\partial X_{p v}} d X_{p v}+\frac{\partial \ln V_{\Phi}}{\partial F e} d F e \\
\mathrm{~d} \ln \rho & =\frac{\partial \ln \rho}{\partial T} d T+\frac{\partial \ln \rho}{\partial X_{p v}} d X_{p v}+\frac{\partial \ln \rho}{\partial F e} d F e
\end{aligned}
$$


Alternatively, one can use the relative anomalies of compressional-wave velocity $\left(\mathrm{d} \ln V_{P}\right)$ instead of $\mathrm{d} \ln V_{\Phi}$. Equations (6) are the basis for a thermo-chemical interpretation of seismic observations. They can either be used by combining two observables (seismic ratios), or be directly inverted for anomalies of temperature and composition.

\section{THERMO-CHEMICAL STRUCTURE: SEISMOLOGICAL EVIDENCES}

\subsection{First hints from seismic ratios}

Several tomographic models based on joint inversion of different seismic data now provide independent constraints on shear and compressive (or bulk sound) velocity anomalies (e.g., Su and Dziewonski, 1997; Kennett et al., 1998; Vasco and Jonhson, 1998; Masters et al., 2000). Seismic ratios combine this information to make a diagnostic of the origin of the observed velocity anomalies. The most commonly used is the ratio of relative shear to compressional velocity anomalies,

$$
R_{P} \equiv \frac{\mathrm{d} \ln V_{S}}{\mathrm{~d} \ln V_{P}} .
$$

Since any scaling $a d T+a d C$ will result in the same value of $R_{P}$, no quantitative information about the amplitude of the variations of temperature $(d T)$ and composition $(d C)$ can be accessed. However, lateral variations of $R_{P}$ provide clear qualitative evidence for compositional variations in the deep mantle (Master et al., 2000; Saltzer et al., 2001). In a previous study (Deschamps and Trampert, 2003), we showed that a full statistical analysis of the histograms of block-byblock values of $R_{P}$ provides useful information on the origin of seismic velocity anomalies.

Figure 5 compares histograms predicted by the global model SB10L18 and synthetic histograms computed for several origins of the velocity anomalies using sensitivities from Figure 4. If only temperature variations contribute to the velocity anomalies, the possible ranges of values of $R_{P}$ are obtained by varying the sensitivities of $V_{S}$ and $V_{P}$ to temperature within their error bars. The results (shaded areas) clearly differ from the observed histograms (red curves), which show a strong dispersion at all depths. Accounting for reasonable errors in the tomographic model (Beghein et al., 2002) increases the dispersion in the distribution of $R_{P}$ (blue curves), but still do not explain the observed dispersion. The observed velocity anomalies can thus not be purely thermal in origin. We also computed distributions of $R_{P}$ for pure compositional effects, including errors in the tomographic models. For perovskite (green curves), the distributions of $R_{P}$ have a similar dispersion than for temperature, but peak at smaller values. For iron (orange curves) they are slightly more dispersed than for temperature, and peak at similar values, suggesting that $R_{P}$ cannot discriminate between temperature and iron variations, hence the need for density information. None of the compositional effects alone can explain 


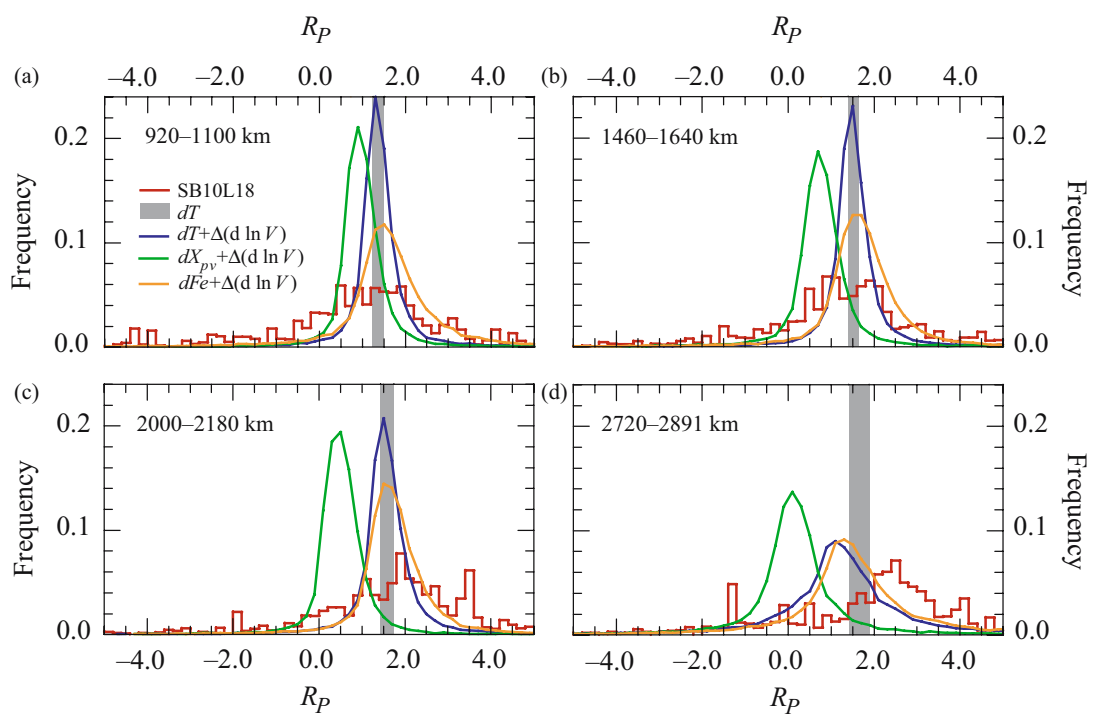

Figure 5. Seismic ratios $R_{P}=\mathrm{d} \ln V_{S} / \mathrm{d} \ln V_{P}$ in the lower mantle. Red curves are block-by-block histograms predicted by SB10L18 (Masters et al., 2000). Dashed areas cover one standard deviation around the mean value obtained for velocity anomalies of a purely thermal origin. Blue curves are obtained by adding simulated errors $\Delta(\mathrm{d} \ln V)$ in the tomographic model to the thermal contribution. Simulated errors have a Gaussian distribution with standard deviation obtained by multiplying the RMS of SB10L18 by the relative error from Beghein et al. (2002). From top to bottom, $\Delta\left(\mathrm{d} \ln V_{S}\right)$ is equal to $2.66 \times 10^{-3}, 1.66 \times 10^{-3}$, $1.93 \times 10^{-3}$, and $5.84 \times 10^{-3}$, and $\Delta\left(\mathrm{d} \ln V_{P}\right)$ is equal to $1.83 \times 10^{-3}, 2.57 \times 10^{-3}, 1.26 \times 10^{-3}$, and $3.12 \times 10^{-3}$. Green and orange curves are obtained by adding simulated errors $\Delta(\mathrm{d} \ln V)$ to the velocity anomalies due to pure perovskite and pure iron variations, respectively.

the observed histograms, but a combination of thermal and compositional effects might.

Ideally, one would like to vary $d T, d X_{p v}$ and $d F e$ within reasonable ranges, compute associated histograms, and compare them with the observed histograms to decide on bounds for $d T, d X_{p v}$ and $d F e$ that fit the observations best. As noted above, however, combinations of $d T, d X_{p v}$ and $d F e$ that differ by a multiplicative factor give the same value of $R_{P}$. While the full histograms of seismic ratios are a good indicator of chemical heterogeneities, a quantitative estimate remains impossible.

\subsection{Deterministic inversions of probabilistic seismic tomography}

Deterministic inversions of shear-wave and compressional-wave (or bulk-sound) velocity have reported lateral variations of composition in the deep mantle (Forte and Mitrovica, 2001; Deschamps and Trampert, 2003), but a close inspection of the possible sources of uncertainties (including the uncertainties in the sensitivities 

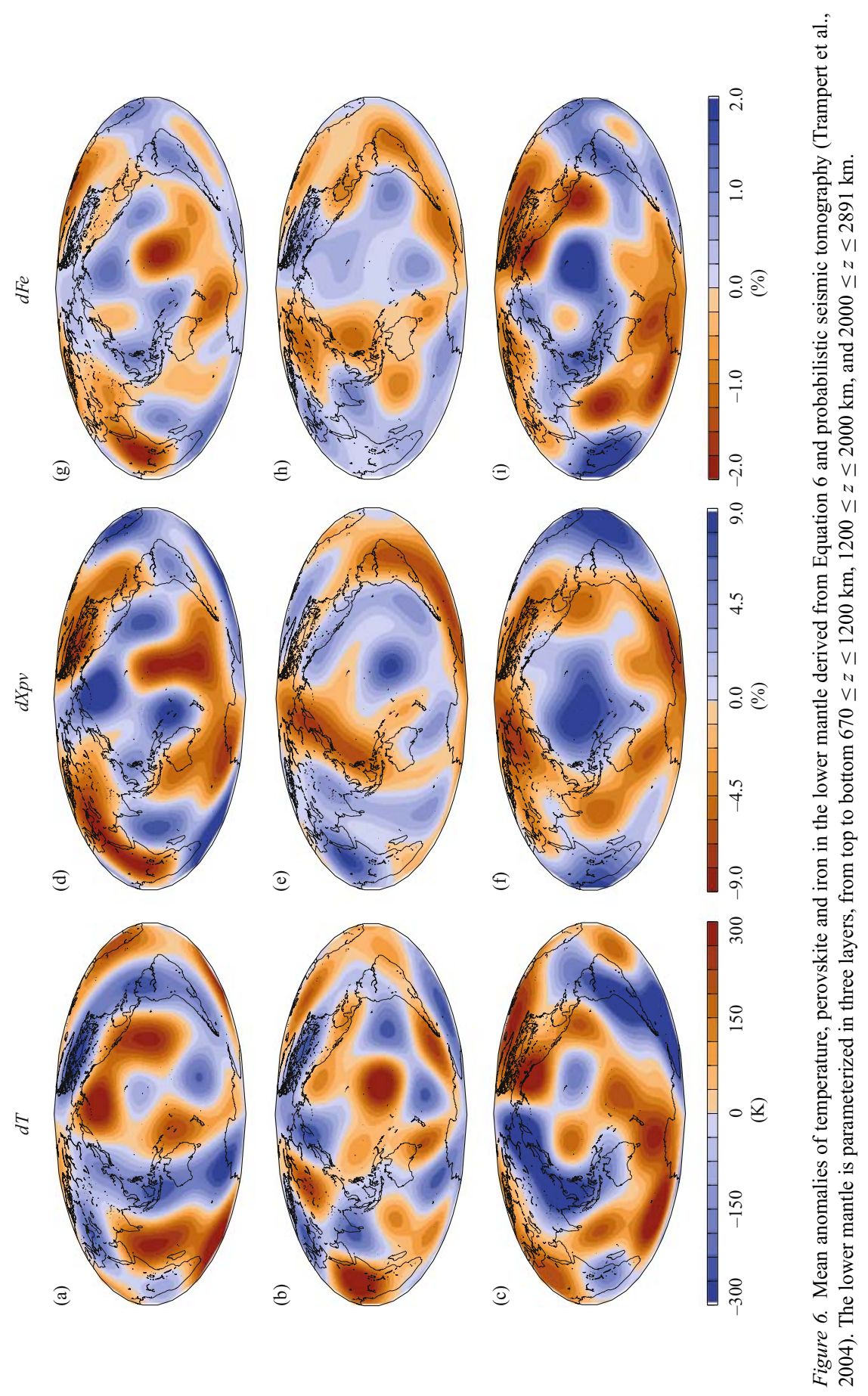
and reasonable uncertainties in the tomographic model) revealed that these variations are not robust (Deschamps and Trampert, 2003) because of a lack of density information.

In a previous paper (Trampert et al., 2004), we inverted the density and seismic velocity anomalies of RT246g for lateral variations of temperature, perovskite and iron. Since seismic observable and sensitivities are probability density functions, we performed a search that returned likelihoods for anomalies of temperature and composition. Assuming the likelihoods to be Gaussian, they can be represented by a mean and a standard deviation. The error bars on temperature and composition account for errors in the data and in the sensitivities. The latter, however, contribute for less than $10 \%$ to the total uncertainties, which are dominated by data errors. The resulting uncertainties are remarkably uniform within each layer (RMS uncertainties are listed in Table 2).

Figure 6 shows the mean of the lateral variations in $d T, d X_{p v}$ and $d F e$ in each layer. Note that, in the deepest layer, the strongest excess in perovskite and iron are found beneath Africa and the Pacific. In the top and mid mantle, amplitudes of temperature and composition anomalies are globally smaller (Table 2). A notable exception are the anomalies of perovskite in the top layer $(670 \leq z \leq$ $1200 \mathrm{~km}$ ), which vary between $-8.5 \%$ and $11.9 \%$ (Fig. $6 \mathrm{~d}$ ). A comparison between Figure 3 and Figure 6 do not show striking similarities between the distributions of $\mathrm{d} \ln V_{S}$ and $d T$. The correlations between $\mathrm{d} \ln V_{S}$ and $d T$ (Table 3) are clearly too low to obtain temperature variations from $\mathrm{d} \ln V_{S}$ scaling. Further comparisons (Table 3 ) suggest that $\mathrm{d} \ln V_{\Phi}$ is an excellent proxy for perovskite variations independent of depth, and that $\mathrm{d} \ln \rho$ maps iron variations very well below $z=1200 \mathrm{~km}$.

A striking result is that low velocities in the deep mantle beneath Africa and the Pacific are due to an enrichment in perovskite and iron, which makes them denser than the surrounding mantle. Because sensitivities of shear-wave velocity to iron

Table 3. Correlation between tomographic observables and thermo-chemical parameters ${ }^{\mathrm{a}}$

\begin{tabular}{lcrrr}
\hline Layer & & $\mathrm{d} \ln V_{S}$ & $\mathrm{~d} \ln V_{\Phi}$ & \multicolumn{1}{c}{$\mathrm{d} \ln \rho$} \\
\hline $670-1200 \mathrm{~km}$ & $d T$ & -0.564 & 0.420 & -0.524 \\
& $d X_{p v}$ & -0.033 & 0.907 & 0.114 \\
& $d F e$ & 0.104 & 0.790 & 0.716 \\
$1200-2000 \mathrm{~km}$ & $d T$ & -0.701 & 0.089 & -0.790 \\
& $\mathrm{~d} X_{p v}$ & -0.332 & 0.889 & -0.281 \\
& $d F e$ & -0.135 & 0.432 & 0.898 \\
$2000-2891 \mathrm{~km}$ & $d T$ & -0.302 & -0.102 & -0.863 \\
& $d X_{p v}$ & -0.792 & 0.933 & 0.277 \\
& $d F e$ & -0.524 & 0.604 & 0.942 \\
\hline
\end{tabular}

${ }^{a}$ Correlations are computed for mean values, on the original grid $15^{\circ} \times 15^{\circ}$. 
(Fig. 4a, light grey area) are negative throughout the lower mantle, this excess in iron results in low $V_{S}$-anomalies and could erroneously be interpreted as a temperature increase.

\section{THERMO-CHEMICAL STRUCTURE: GEODYNAMICAL CONSEQUENCES}

\subsection{Observed variations of density}

Trampert et al. (2004) clearly demonstrated that strong lateral variations of composition are present throughout the lowermost mantle. It is crucial, from a dynamical point of view, to separate and compare chemical $\left(\mathrm{d} \ln \rho_{c}\right)$ and thermal $\left(\mathrm{d} \ln \rho_{T}\right)$ contributions to the density anomalies. At each model point, we randomly sampled the anomalies of temperature and composition (perovskite and iron) within their Gaussian distributions. We similarly sampled the sensitivities of density and generated 20 million models of $\mathrm{d} \ln \rho_{T}$ and $\mathrm{d} \ln \rho_{c}$ density. Figure 7 shows the average maps of $\mathrm{d} \ln \rho_{T}$ and $\mathrm{d} \ln \rho_{c}$ in each layer. Again, uncertainties (defined as one standard deviation) are remarkably homogeneous within each layer (RMS of these uncertainties are given in Table 2).

In the bottom layer, $\mathrm{d} \ln \rho_{T}$ is small, and varies between -0.31 and $0.44 \%$ (Fig. $7 \mathrm{c}$ ). As previously noted, the small values of $\mathrm{d} \ln \rho_{T}$ are due to the decrease of the thermal expansion at great depths (Fig. 4c, dashed area). In comparison, $\mathrm{d} \ln \rho_{c}$ are on average twice as large (Fig. $7 \mathrm{f}$ ), and vary between $-0.71 \%$ and $0.95 \%$. Due to the small values of the sensitivity of density to perovskite (Fig. 4c, light grey area), variations in perovskite contribute less than $10 \%$ to the total $\mathrm{d} \ln \rho_{c}$. The chemical density anomalies are therefore dominated by iron anomalies, as also suggested by the high correlation between the relative density anomalies and the iron anomalies (Table 3 ). Excess in iron increases the density up to $1.0 \%$, the largest anomalies being observed beneath Africa and the Pacific. Since thermal density anomalies in these regions are of the order of $0.2 \%$, the material is not buoyant. Power spectra of $\mathrm{d} \ln \rho_{T}$ and $\mathrm{d} \ln \rho_{c}$ reveal that each degree of the spherical harmonic expansion is dominated by chemical density anomalies (Fig. 8c). Moreover, most of the chemical and thermal signals are contained in degree 2. Degrees 4 and 6 are weaker than degree 2 by a factor 4 .

Although weaker than in the bottom layer, the chemical density anomalies in the intermediate layer $(1200 \leq z \leq 2000 \mathrm{~km})$ are of the same order as the thermal density anomalies, and are dominated by degree 2 . In general, this layer has small anomalies of temperature and composition.

Thermal and chemical buoyancies become stronger at the top of the lower mantle $(670 \leq z \leq 1200 \mathrm{~km}) . \mathrm{d} \ln \rho_{T}$ varies between $-0.64 \%$ and $0.57 \%$, and is stronger, on average, than in the bottom layer. Degree 2 is slightly stronger than in the lowermost layer, but, interestingly, there is more power in the degree 4 than in the degree 2 (Fig. 8a). $\mathrm{d} \ln \rho_{c}$ is not as strong as in the lowermost layer, but still varies between $-0.80 \%$ and $0.67 \%$. Most of the difference in amplitude is due to the degree 2 , which 


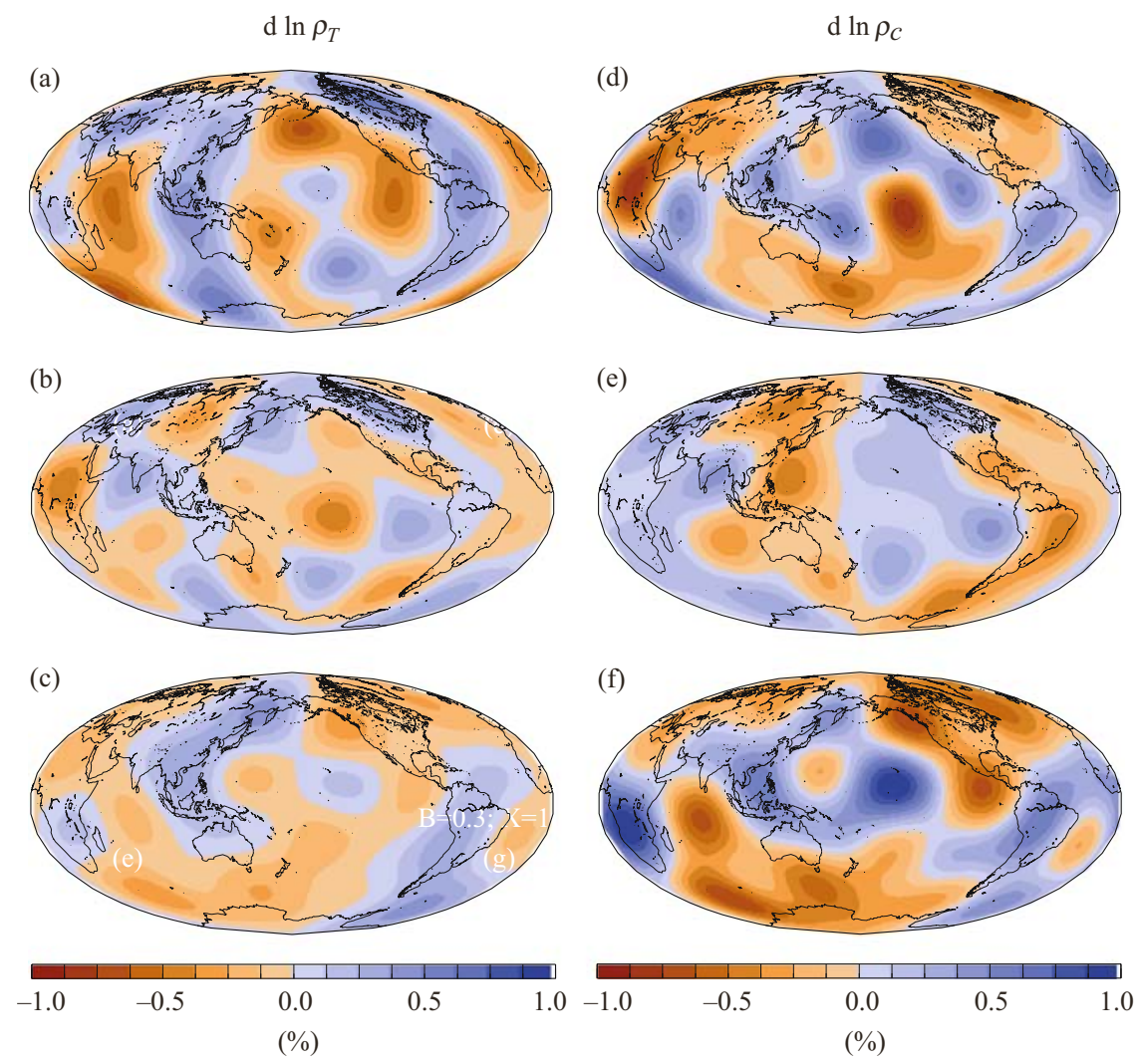

Figure 7. Thermal $(\mathrm{a}-\mathrm{c})$ and chemical $(\mathrm{d}-\mathrm{f})$ contributions to the relative anomalies of density in the lower mantle. The lower mantle is parameterized in three layers, from top to bottom $670 \leq z \leq 1200 \mathrm{~km}$, $1200 \leq z \leq 2000 \mathrm{~km}$, and $2000 \leq z \leq 2891 \mathrm{~km}$.

is half of that the bottom layer. Note that the anomalies of perovskite now make up $30 \%$ of the total $\mathrm{d} \ln \rho_{c}$.

\subsection{Comparison with thermo-chemical convection}

The numerical models of convection we used are defined on Cartesian grids (see Section 2) and can therefore be transferred to the Fourier domain. To compare them with global tomographic models, which are expanded in spherical harmonics, one must carefully compute the power spectra for each expansion. We did this using a method developed by Chevrot et al. (1998). From the convection models, we first performed a Fourier transform of the $\mathrm{d} \ln \rho_{T}$ and $\mathrm{d} \ln \rho_{c}$ at each depth, and computed the signal contained in each wave number $k=\sqrt{k_{x}^{2}+k_{y}^{2}}$, where $k_{x}$ and $k_{y}$ are the 

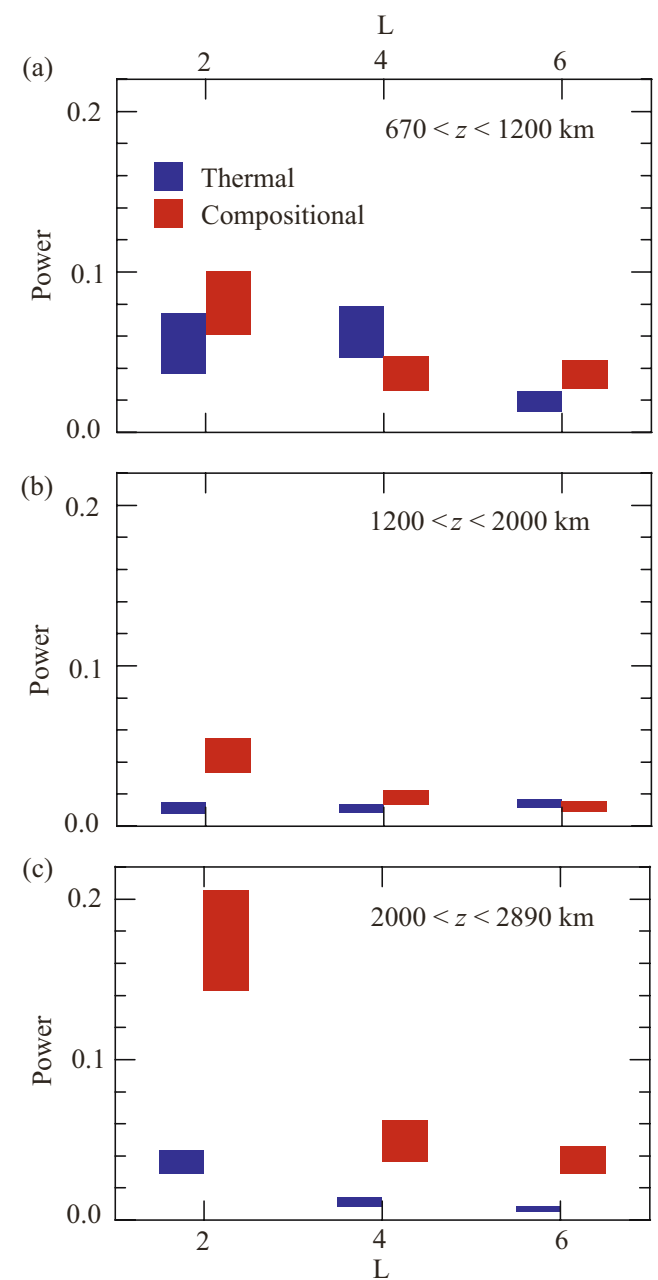

Figure 8. Power spectra of thermal (blue histograms) and chemical (red histograms) density anomalies predicted by probabilistic tomography model RT246g (Trampert et al., 2004) for degrees 2,4 and 6 . Each histogram covers one standard deviation around the mean value of the power.

wave numbers along the $x$ - and $y$-axis. The signal contained in the spherical harmonic degree $\ell$ is equal to the sum of the signals contained in the wave numbers $k$ that satisfy $(\ell+1 / 2) \leq k a \leq(\ell+3 / 2)($ Chevrot et al., 1998):

$$
P(\ell)=\frac{2 \pi}{N_{x}^{2} N_{y}^{2}(\ell+1 / 2)} \sum_{(\ell+1 / 2) \leq k a \leq(\ell+3 / 2)}|\mathrm{d} \ln \rho(k)|^{2},
$$


where $a$ is the Earth's radius, and $N_{x}$ and $N_{y}$ the number of grid points in $x$ and $y$ directions. The tomographic model RT246g represents the lower mantle in three layers, and contains spherical harmonic degrees 2, 4 and 6 only, whereas the numerical models of Tackley (2002) sample the lower mantle with 50 points, and contains signal up to degree 32. To make meaningful comparisons, we averaged the synthetic $\mathrm{d} \ln \rho_{T}$ and $\mathrm{d} \ln \rho_{c}$ (Eqs. 4-5) in comparable layers as defined by RT246g, and filtered them for spherical harmonic degrees 2, 4 and 6.

The RMS of $\mathrm{d} \ln \rho_{T}$ and $\mathrm{d} \ln \rho_{c}$ from seismology and convection models are plotted in Figure 9. All the numerical models we considered predicted the RMS of $d \ln \rho_{T}$ and $\mathrm{d} \ln \rho_{c}$ that fitted within one standard deviation of the observed RMS. The best fit is obtained for $B=0.2, X=10 \%$. In the intermediate layer, the RMS of $\mathrm{d} \ln \rho_{c}$ for the cases $B=0.3, X=10 \%$, and $B=0.4, X=10 \%$ are close to the observed lower bound, but cannot be excluded. Analysis of the RMS is therefore not a conclusive test, at least with the current depth parameterization of probabilistic seismic tomography. Models with finer parameterization will certainly provide a better test, but are not yet available. Clearer conclusions can be drawn from comparing the power spectra of observed and synthetic distributions (Fig. 10). In case of a stable layer of dense material ( $B=0.3, X=30 \%$, green histograms), the strongest chemical density anomalies are found between the intermediate and lower layers (Fig. 1d). As a result, spectra of $\mathrm{d} \ln \rho_{c}$ in the bottom and intermediate layers are similar one another, which is not observed by RT246g. In particular, degree 2 has too little power in the bottom
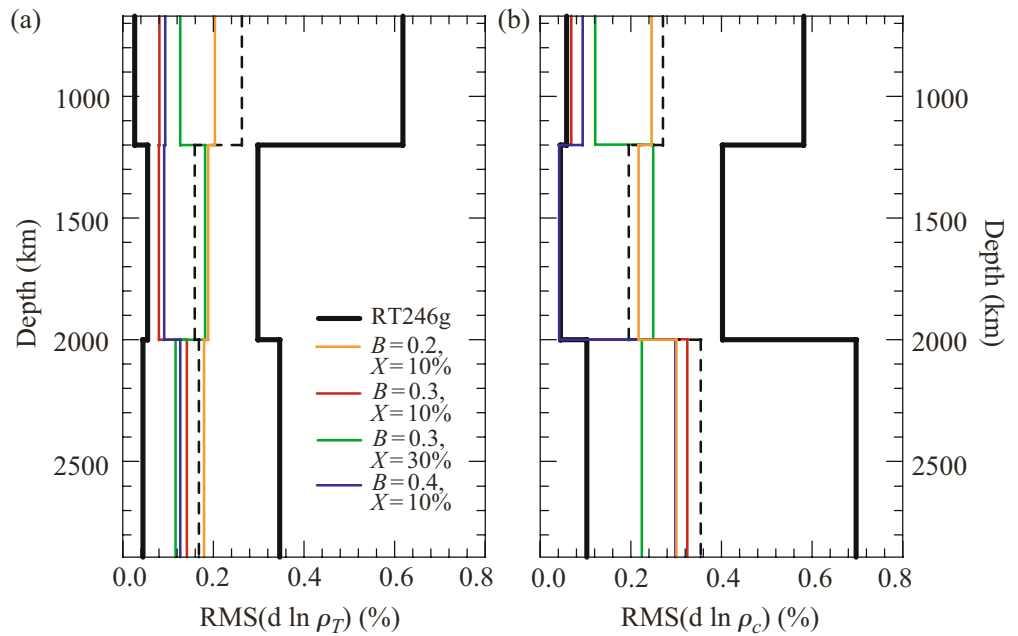

Figure 9. RMS of relative density anomalies due to (a) thermal and (b) compositional effects. The thin dashed curve represents the RMS of the average observed models (Fig. 7), and the thick curves represent de RMS of the models with one standard deviation around these models. Colored curves represent the RMS predicted by 3-D Cartesian numerical models of thermo-chemical convection (Tackley, 2002) with several buoyancy ratio $(B)$ and volumetric fraction of dense material $(X)$. Density anomalies from numerical models of convection are filtered for degrees 2,4 , and 6 . 

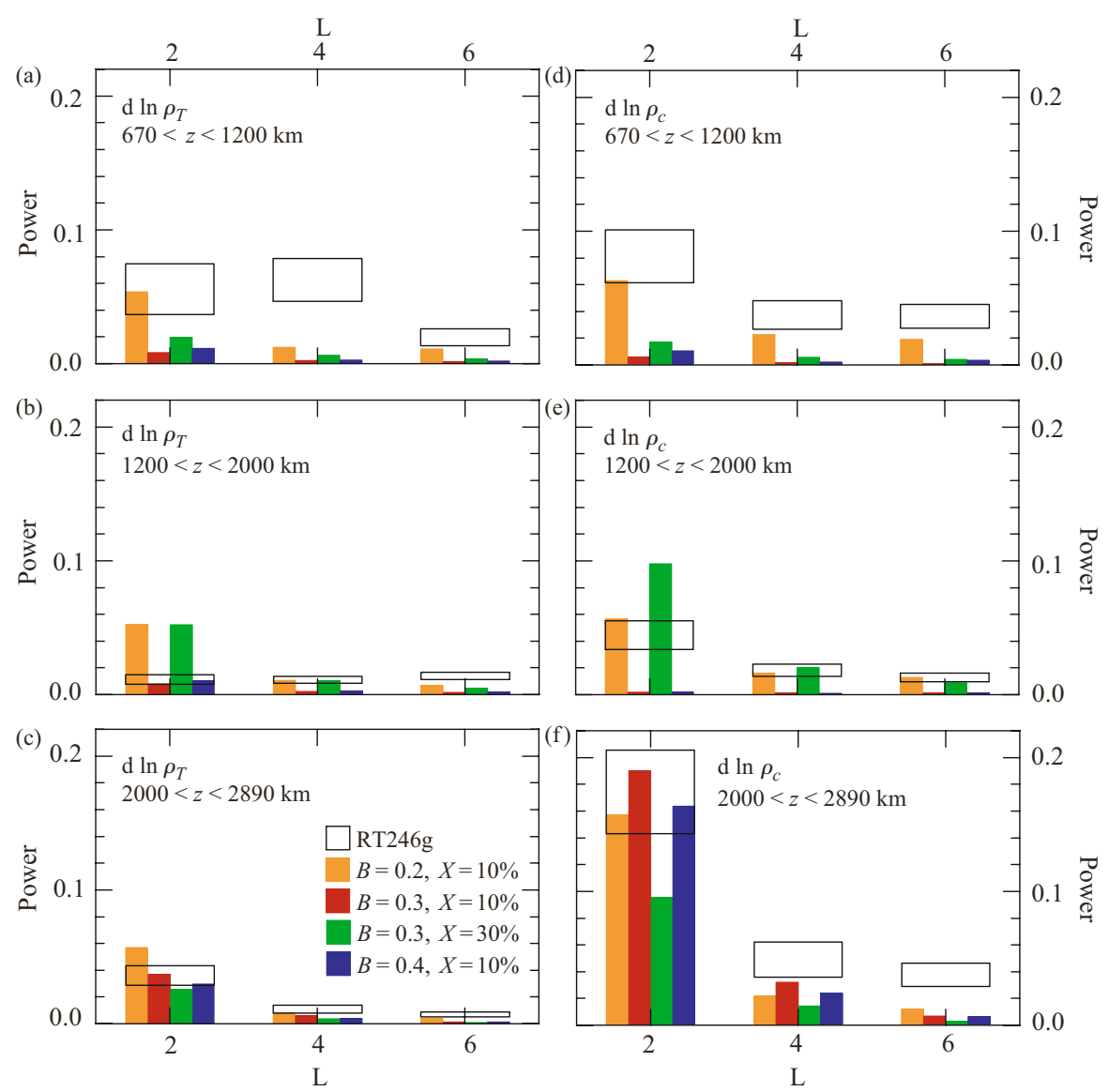

Figure 10. Power spectra of thermal and chemical contributions to the density anomalies derived from numerical models of thermo-chemical convection (Tackley, 1998, 2002), for degrees 2, 4 and 6, and for several buoyancy ratio $(B)$ and volumetric fraction of dense material $(C)$. Because numerical models are defined on a 3-D Cartesian grid, we used the method of Chevrot et al. (1998) to compute their spherical harmonic coefficients (see text). For comparison, the white rectangles cover one standard deviation around the means of the observed power (see Fig. 8).

layer, and too much in the intermediate layer. This case also fails to explain the observed power spectrum of $\mathrm{d} \ln \rho_{T}$ and $\mathrm{d} \ln \rho_{c}$ in the upper layer, and the degree 2 of $\mathrm{d} \ln \rho_{T}$ in the intermediate layer. The thin stable layer $(B=0.4, X=10 \%$, blue histograms) and discontinuous spoke pattern $(B=0.3, X=10 \%$, red histograms $)$ show strong lateral variations of temperature and composition below $2000 \mathrm{~km}$, but not at shallower depths. They explain the observed power spectra in the bottom layer, but not in the intermediate (except the degree 2 of $\mathrm{d} \ln \rho_{T}$ ) and top layers. Finally, the case $B=0.2, X=10 \%$ (orange histograms) explains most of the power spectra 
of $d \ln \rho_{c}$, at least within two standard deviations (95\% certainty). It fits $d \ln \rho_{T}$ less well, most of the discrepancies being due to the degree 2 in the intermediate layer, and to the degree 4 in the top layer.

The previous comparisons suggest that the model with $B=0.2$ gives the best agreement to the observed density anomalies. As an additional test, we estimated the global buoyancy ratio associated with the observed $d \ln \rho_{T}$ and $\mathrm{d} \ln \rho_{c}$. We considered that the iron fraction of the regular material is equal to $11 \%$, and that the dense material is enriched in iron by $2.0-2.5 \%$, as reported in Section 5 . If the mantle aggregate consists in $80 \%$ perovskite and $20 \%$ magnesio-wüstite, the density and thermal expansion at the surface are $\rho_{s}=4240 \mathrm{~kg} / \mathrm{m}^{3}$ and $\alpha_{s}=2 \times 10^{-5} \mathrm{~K}^{-1}$, and the chemical density contrast due to the enrichment in iron varies between $\Delta \rho_{c}^{\max }=$ $40 \mathrm{~kg} / \mathrm{m}^{3}$ and $\Delta \rho_{c}^{\max }=49 \mathrm{~kg} / \mathrm{m}^{3}$ (Deschamps and Trampert, 2004). Taking $\Delta T_{S}=$ $2500 \mathrm{~K}$, the thermal density contrast is $\Delta \rho_{T}^{\max }=213 \mathrm{~kg} / \mathrm{m}^{3}$, and the buoyancy ratio varies between $B=0.19$ and $B=0.23$.

\section{DISCUSSION}

The low shear wave velocities observed in the deep mantle below Africa and the Pacific are fully explained by long wavelength lateral variations of composition. Iron increases are responsible for strong (up to 1\%) anomalies of density, and variations in perovskite are significant (up to $9 \%$ ) and explain bulk-sound velocity very well. The comparisons performed in the previous section, however, suggest that these variations are not large enough to maintain a stable chemical layering. If a dense layer is present it must be unstable, i.e., the domes or piles of dense material are generated in the bottom of the mantle and oscillate or are entrained upwards. The current parameterization of probabilistic seismic tomography, however, cannot resolve these structures.

A question that is not addressed in this paper is the origin of the compositional variations. We easily identify three different scenarios. First, early differentiation may have created a dense layer at the bottom of the mantle. Because the buoyancy ratio is not large enough $(B<0.3)$, this layer is unstable, and the material is entrained upwards. A major disadvantage of this scenario is that the layer must have been rapidly entrained and mixed in the mantle, unless it is regularly fed with new material. Gonnerman et al. (2002) showed that even if the initial layer is stable, entrainment of material would progressively decrease the density contrast and lead to a present unstable layer. Second, slabs may penetrate in the lower mantle and reach the CMB, where they sediment in pools (e.g., Christensen and Hofmann, 1994). Again, the density contrast and quantity of sinking material should not be too large to allow the development of instabilities. Slabs could temporarily stack in the mid-mantle, and reach the bottom of the mantle in occasional avalanches (Tackley et al., 1994). There are indeed some indications from classical tomography that significant amounts of slabs are deflected in the top of the lower mantle, around $1000 \mathrm{~km}$ (Fukao et al., 2001). The variations of temperature and composition (mainly perovskite) observed by probabilistic tomography at the top of the lower mantle could also be due to 
deflected slabs. Finally, iron excess may result from percolation from the core (e.g., Knittle and Jeanloz, 1991). A recent study (Humayun et al., 2004) suggests that the lower mantle beneath Hawaii is enriched in iron by $1-2 \%$. Interestingly, the largest iron anomalies we found are close to $2 \%$, and are located beneath Hawaii (Fig. 6i). The actual influence of iron percolation from the core remains however difficult to estimate. These scenarios are end-members, and a mix of them is more likely required to describe the Earth's mantle. Discriminating between the previous scenarios (or a mix of them) is not possible from the results presented in this paper only, but needs to integrate other types of information, in particular geochemical constraints.

The thermo-chemical distributions from convection we have tested are snapshots of time-dependent calculations, which do not necessarily correspond to the present state of the mantle. A full comparison requires to test series of snapshots at different times. For $B=0.3, X=30 \%$, the layer of dense material remains stable for a long period of time ( $3 \mathrm{~Gy}$ ), with similar spectral signature. The spokes pattern $(B=0.3, X=10 \%)$ and thin dense layer $(B=0.4, X=10 \%)$ modes are also stable over long periods of time (5 Gy and more). For $B=0.2, X=10 \%$, mature piles (such as those plotted in Fig. 1) develop in about 200 My. Once they are formed, piles feed the mantle with dense material until the fluid is chemically homogeneous. Spectral signatures for early stages do not explain the observation as well as mature piles.

Hotspots are a major ingredient of mantle dynamics, but their origin is still debated. Recent tomographic studies (Zhao, 2001, 2004; Montelli et al., 2004) image hotspot tails down to various depths. Several hotspots, including Hawaii, seem to originate in the lowermost mantle, close to the CMB. The resolution of RT246g does not allow detailed inferences on the origin of hotspots, but maps of density anomalies (Fig. 3g-i) clearly show that deep-rooted hotspots, as defined by and Courtillot et al. (2003) and Montelli et al. (2004), do not systematically plot continuously on buoyant material throughout the lower mantle. Within error bars in density anomalies, only one third of these deep hotspots correlate with negative density anomalies in the lowermost mantle, and only two (Iceland and Azores) plot on buoyant material throughout the mantle. It is important to keep in mind that Zhao $(2001,2004)$ and Montelli et al. (2004) map compressional seismic velocity anomalies only, but not density. Neglecting compositional variations, they assume that the seismic velocity anomalies are purely thermal in origin, i.e., that low velocity regions are related to hot, hence buoyant material. As shown in this chapter and elsewhere (Trampert et al., 2004), chemically dense material dominates thermal buoyancy in the lower mantle, and still results in low velocities. For instance, in the lowermost mantle, the Hawaiian region is associated with dense, non-buoyant material (Fig. 3i) and low shear-wave velocities (Fig. 3c).

The thermo-chemical structure we inferred is based on a parameterization of composition that consists in perovskite and iron variations. Recent developments in mineral physics, which are not accounted for in this study, may complicate the interpretation of seismic information, and modify our inferences. Murakami et al. 
(2004) reported a new phase transition from perovskite to post-perovskite around $2700 \mathrm{~km}$. We did preliminary tests using available thermo-elastic data for postperovskite (Tsuchiya et al., 2004a,b), but we did not find any dramatic differences compared to the sensitivities plotted in Figure 4. The biggest change is for the sensitivities of density to perovskite, which are positive from the depth of the transition down to the CMB. The amplitudes of the sensitivities, however, remain small. Thus, the phase transition to post-perovskite should not dramatically modify the thermo-chemical structure inferred from probabilistic tomography. Still, it may have significant consequences on the lower mantle dynamics (Nakagawa and Tackley, 2005). Interestingly, the large value of its Clapeyron slope (around 8$10 \mathrm{MPa} / \mathrm{K}$ ) could explain the good correlation (equal to 0.7 ) between the observed thermal and compositional contributions to density at the bottom of the mantle, which is not predicted by the models of thermo-chemical convection we used. In cold regions (e.g., slabs) perovskite may transform to post-perovskite at shallower depths than in hot regions (if they transform at all before the CMB). In addition, there are indications that Fe-perovskite may transform at shallower depths than Mg-perovskite (Spera et al., 2006). In that case, the chemically dense and cold regions observed in the lowermost mantle would correspond to iron-rich post-perovskite.

Due to heavy computational requirements, the vertical parmeterization of RT246g is limited to three layers in the lower mantle, which reduces the effects of a postperovskite phase on this study. Further, averaging models of thermo-chemical convection in these layers, we lose important information (compare Figs. $2 b$ and $2 d$ against Fig. 9). A careful statistical analysis, however, allows robust conclusions concerning the lower mantle structure. A thick stable layer of dense material, for instance, implies that the chemical signatures in the mid-mantle $(1200 \leq z \leq 2000 \mathrm{~km})$ and in the lowermost mantle $(2000 \leq z \leq 2891 \mathrm{~km})$ are comparable. On the contrary, a thin stable layer would result in no notable chemical signal down to $2000 \mathrm{~km}$. Since none of these features fit within the observed error bars (Fig. 10), a stable layer of dense material is unlikely to be present at the bottom of the mantle. Probabilistic models of seismic tomography with a better vertical parameterization should be available in the near future by performing more efficient parallel calculations. A more serious problem is that RT246g does not include odd degrees, which potentially contain important information. Including odd spherical harmonic degrees is a difficult task because normal mode splitting cannot access these degrees very easily. A last point is that a finer lateral resolution, including degrees higher than 6 , is not so essential to correctly understand the lower mantle. The power spectrum of relative density anomalies predicted by models of thermo-chemical convection strongly decreases for degrees higher than 7, as shown in Figure 11 for the case $B=0.2$ and $X=10 \%$. The power spectra for other models of thermo-chemical convection (not shown here), have a similar decrease. If mantle convection is similar to one of these models, we would expect that degrees higher than 7 are weak, and do not significantly participate to the nature of tomographic models. This is confirmed by recent tomographic models (Gu et al., 2001). 

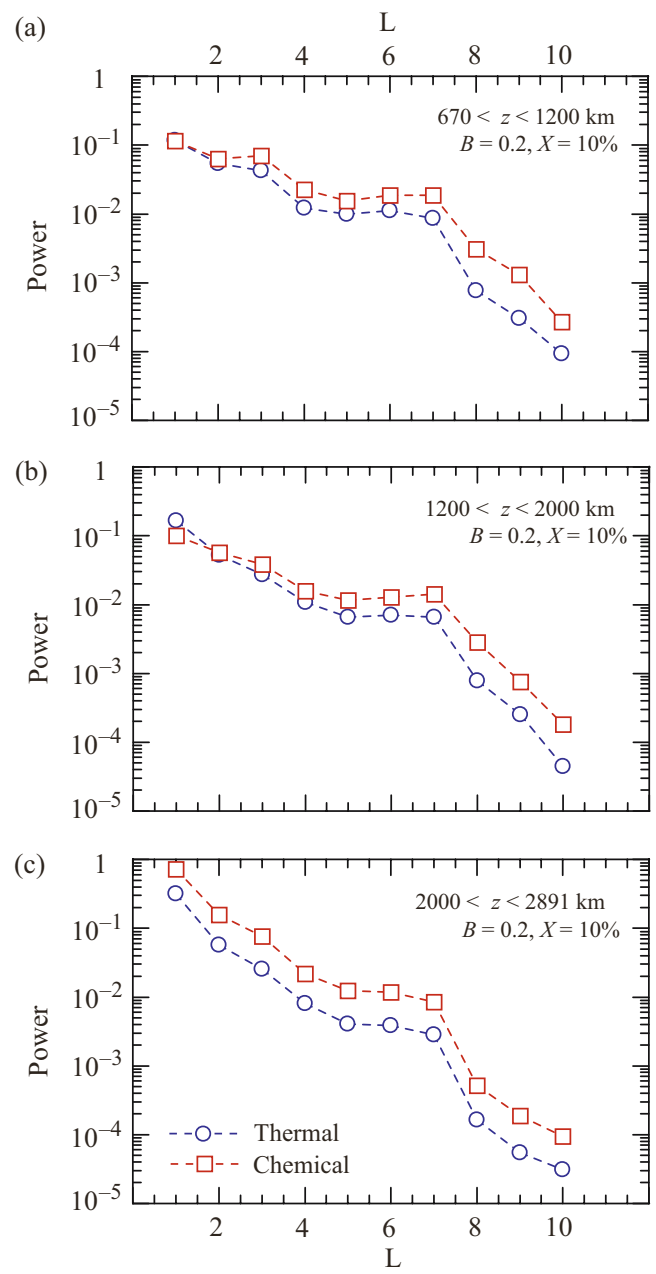

Figure 11. Power spectrum of thermal (blue curves) and chemical (red curves) contributions to the density anomalies predicted by a 3-D Cartesian numerical model of thermo-chemical convection (Tackley, 2002) with buoyancy ratio $B=0.2$, and volumetric fraction of dense material $X=10 \%$. The power in each spherical harmonic degree is calculated following Chevrot et al. (1998). Degrees $\ell=1-10$ are represented, and the signal is averaged within each layer defined by the probabilistic model of seismic tomography RT246g.

\section{CONCLUSION}

Our view of mantle structure and dynamics has rapidly changed during the last decade. A strong indication of dense material (Ishii and Tromp, 1999) or chemical variations in the lower mantle (Forte and Mitrovica, 2001; Saltzer et al., 2001) has now turned 
to robust quantitative estimates of the variations of temperature and composition (Trampert et al., 2004). Chemical heterogeneities are clearly present throughout the mantle. In the top layer $(670 \leq z \leq 1200 \mathrm{~km})$, thermal and chemical signatures could be related to slab deflection. In the lowermost mantle (2000 $\leq z \leq 2891 \mathrm{~km})$ strong anomalies of iron, whose origin is still to be determined, are present in regions usually referred to as "superplumes". From a geodynamical point of view, the presence of lateral variations of composition is observed in recent models of thermo-chemical convection, which now account for fluid compressibility. Robust comparisons between geophysical observations, mainly seismology, and geodynamical models can now be attempted in order to discriminate between certain modes of convection in the mantle. Preliminary results discussed in this chapter indicate that a stable layer of dense material is unlikely to be present at the bottom of the mantle. Piles of dense material entrained upwards from a dense, unstable layer statistically explain probabilistic tomography better, but discrepancies remain in the top of the lower mantle, possibly linked to the phase transformation around $670 \mathrm{~km}$ and/or slabs deflection around $1000 \mathrm{~km}$. Future researches should focus on estimating the influence of recent mineral physics findings on lower mantle seismic velocity and density, exploring and testing scenarios that can produce strong lateral variations of iron at the bottom of the mantle, computing probabilistic models of tomography with finer vertical parameterization, and testing models of thermo-chemical convection that include more complexities, such as a phase transformation at $670 \mathrm{~km}$, or the post-perovskite phase transition in the lowermost mantle.

\section{ACKNOWLEDGEMENTS}

We appreciated the constructive reviews of an anonymous reviewer. This research was funded by the Netherlands Research Center for Integrated Solid Earth Sciences (ISES) and Utrecht University.

\section{REFERENCES}

Anderson, O.L. (1995) Equations of State of Solids for Geophysics and Ceramic Sciences, Oxford University Press, Oxford, 405pp.

Anderson, D.L. (2001) Top-down tectonics. Science, 293, 2016-2018.

Beghein, C., J.S. Resovsky, and J. Trampert (2002) P and S tomography using normal-mode and surface waves data with a neighbourhood algorithm. Geophys. J. Int., 149, 646-658.

Chevrot, S., J.P. Montagner, and R. Snieder (1998) The spectrum of tomographic Earth models. Geophys. J. Int., 33, 783-788.

Christensen, U.R., and A. Hofmann (1994) Segregation of subducted oceanic crust in the convecting mantle. J. Geophys. Res., 99, 19867-19884.

Courtillot, V., A. Davaille, J. Besse, and J. Stock (2003) Three distinct types of hotspots in the Earth's mantle. Earth Planet. Sci. Lett., 205, 295-308.

Daniel, I., J.D. Bass, G. Fiquet, H. Cardon, J. Zhang, and M. Hanfland (2004) Effect of aluminium on the compressibility of silicate perovskite. Geophys. Res. Lett., 31, doi: 10.1029/2004GL020213.

Davaille, A. (1999) Simultaneous generation of hotspots and superswells by convection in a heterogeneous planetary mantle. Nature, 402, 756-760. 
Deschamps, F., and J. Trampert (2003) Mantle tomography and its relation to temperature and composition. Phys. Earth Planet. Inter, 140, 277-291.

Deschamps, F., and J. Trampert (2004) Towards a lower mantle reference temperature and composition. Earth Planet. Sci. Lett., 222, 161-175.

Forte, A.M., and J.X. Mitrovica (2001) Deep-mantle high-viscosity flow and thermo-chemical structure inferred from seismic and geodynamic data. Nature, 410, 1049-1056.

Fukao, Y., S. Widiyantoro, and M. Obayashi (2001) Stagnant slabs in the upper and lower mantle transition region. Rev. Geophys., 39, 291-323.

Goes, S., R. Govers, and P. Vacher (2000) Shallow mantle temperature under Europe from P and S wave tomography. J. Geophys. Res., 105, 11153-11169.

Gonnerman, H.M., M. Manga, and A.M. Jellinek (2002) Dynamics and longevity of an initially stratified mantle. Geophys. Res. Lett., 29, doi:10.1029/2002GL014851.

Gu, Y.J., A.M. Dziewonski, W.J. Su, and G. Ekstrom (2001) Models of the mantle shear velocity and discontinuities in the pattern of lateral heterogeneities. J. Geophys. Res., 106, 11169-11199.

Hansen, U., and D.A. Yuen (1988) Numerical simulations of thermo-chemical instabilities at the coremantle boundary. Nature, 334, 237-240.

Humayun, M., L. Qin, and M.D. Norman (2004) Geochemical evidence for excess iron in the lower mantle beneath Hawai. Science, 306, 91-94.

Ishii, M., and J. Tromp (1999) Normal-mode and free-air gravity constraints on lateral variations in velocity and density of Earth's mantle. Science, 285, 1231-1236.

Jackson, I. (1998) Elasticity, composition and temperature of the earth's lower mantle: A reappraisal. Geophys. J. Int., 134, 291-311.

Jackson, J.M., J. Zhang, and J.D. Bass (2004) Sound velocities and elasticity of aluminous $\mathrm{MgSiO}_{3}$ perovskite: Implications for aluminium heterogeneity in Earth's lower mantle. Geophys. Res. Lett., 31, doi: 10.1029/2004GL019918.

Jeanloz, R., and E. Knittle (1989) Density and composition of the lower mantle. Philos. Trans. R. Soc. Lond. A, 328, 377-389.

Karato, S.I., and B.B. Karki (2001) Origin of lateral variation of seismic wave velocities and density in the deep mantle. J. Geophys. Res., 106, 21771-21783.

Kellogg, L.H., B.H. Hager, and R.D. van der Hilst (1999) Compositional stratification in the deep mantle. Science, 283, 1881-1884.

Kennett, B.L.N., S. Widiyantoro, and R.D. van der Hilst (1998) Joint seismic tomography for bulk sound and shear wave speed in the Earth's mantle. J. Geophys. Res., 103, 12469-12493.

Kiefer, B., L. Stixrude, and R.M. Wentzcovitch (2002) Elasticity of (Mg,Fe) $\mathrm{SiO}_{3}$ perovskite at highpressures. Geophys. Res. Lett., 29, doi: 10.1029/2002GL014683.

Knittle, E., and R. Jeanloz (1991) Earth's core-mantle boundary: Results of experiments at high pressures and temperatures. Science, 251, 1438-1443.

Kung, J., B. Li, D.J. Weidner, J. Zang, and R.C. Liebermann (2002) Elasticity of $\left(\mathrm{Mg}_{0.83}, \mathrm{Fe}_{0.17}\right) \mathrm{O}$ ferropericlase at high pressure: Ultrasonic measurements in conjunction with X-radiation techniques. Earth Planet. Sci. Lett., 203, 557-566.

Masters G., G. Laske, H. Bolton, and A.M. Dziewonski (2000) The relative behavior of shear velocity, bulk sound speed, and compressional velocity, in the mantle: Implications for chemical and thermal structure. In Karato, S.I. et al. (eds.) Earth's Deep Interior: Mineral Physics and Tomography from the Atomic to the Global Scale, Geophys. Mon. Ser., 117, pp. 63-87.

Montelli, R., G. Nolet, F.A. Dahlen, G. Masters, E.R. Engdahl, and S.H. Hung (2004) Finite frequency tomography reveals a variety of plumes in the mantle. Science, 303, 338-343.

Murakami, M., K. Hirose, K. Kawamura, N. Sata, and Y. Ohishi (2004) Post-perovskite phase transition in $\mathrm{MgSiO}_{3}$. Science, 304, 855-858.

Nakagawa, T., and P.J. Tackley (2005) The interaction between the post-perovskite phase change and a thermo-chemical boundary layer near the core-mantle boundary. Earth Planet. Sci. Lett., 238, 204-216.

Olson, P., and C. Kinkaid (1991) Experiment on the interaction of thermal convection and compositional layering at the base of the mantle. J. Geophys. Res., 96, 4347-4354. 
Resovsky, J.S., and J. Trampert (2003) Using probabilistic seismic tomography to test mantle velocitydensity relationships. Earth Planet. Sci. Lett., 215, 121-134.

Romanowicz, B., and Y. Gung (2002) Superplumes from the core-mantle boundary to the lithosphere: Implications for heat flux. Science, 296, 513-516.

Saltzer, R.L., R.D. van der Hilst, H. Kárason (2001) Comparing P and S wave heterogeneity in the mantle. Geophys. Res. Lett., 28, 1335-1338.

Sambridge, M. (1999a) Geophysical inversion with a neighbourhood algorithm — I. Searching a parameter space. Geophys. J. Int., 138, 479-494.

Sambridge, M. (1999b) Geophysical inversion with a neighbourhood algorithm-II. Apraising the ensemble. Geophys. J. Int., 138, 727-746.

Schott, B., and D.A. Yuen (2004) Influences of dissipation and rheology on mantle plumes coming from the D"-layer. Phys. Earth Planet. Inter, 146, 139-145.

Spera, F.J., D.A. Yuen, and G. Giles (2006) Tradeoffs in chemical and thermal variations in the postperovskite phase transition: Mixed phase regions in the deep lower mantle? Phys. Earth Planet. Inter., $159,234-246$.

Su, W.J., and A.M. Dziewonski (1997) Simultaneous inversion of 3D variations in the shear and bulk velocity in the mantle. Phys. Earth Planet. Inter., 100, 135-156.

Tackley, P.J. (1998) Three-dimensional simulations of mantle convection with a thermo-chemical CMB boundary layer: $\mathrm{D}^{\prime \prime}$ ? In Gurnis $\mathrm{M}$. et al. (eds.) The Core-Mantle Boundary Region, Geodynamical Ser., 28, pp. 231-253.

Tackley, P.J. (2002) Strong heterogeneity caused by deep mantle layering. Geochem. Geophys. Geosys., 3, doi: 10.1029/2001GC000167.

Tackley, P.J., D.J. Stevenson, G.A. Glatzmaier, and G. Schubert (1994) Effects of multiple phase transition in a three-dimensional spherical model of convection in Earth's mantle. J. Geophys. Res., 99, $15877-15902$.

Trampert, J., P. Vacher, and N. Vlaar (2001) Sensitivities of seismic velocities to temperature, pressure and composition in the lower mantle. Phys. Earth Planet. Inter., 124, 255-267.

Trampert, J., F. Deschamps, J.S. Resovsky, and D.A. Yuen (2004) Probabilistic tomography maps significant chemical heterogeneities in the lower mantle. Science, 306, 853-856.

Trampert, J., and R.D. van der Hilst (2005) Quantitative interpretation of global seismic tomography. In van der Hilst et al. (eds) Earth's Deep Mantle: Structure, Composition, and Evolution, Geophys Mon. Ser., 160, pp. 47-62.

Tsuchiya, T., J. Tsuchiya, K. Umemoto, and R.M. Wentzcovitch (2004a) Phase transition in $\mathrm{MgSiO}_{3}$ perovskite in the Earth's lower mantle. Earth Planet. Sci. Lett., 224, 241-248.

Tsuchiya T., J. Tsuchiya, K. Umemoto, and R.M. Wentzcovitch (2004b) Elasticity of post-perovskite $\mathrm{MgSiO}_{3}$. Geophys. Res. Lett., 31, doi: 10.1029/2004GL020278.

Vasco, D.W., and L.R. Johnson (1998) Whole Earth structure estimated from seismic arrival times. J. Geophys. Res., 103, 2633-2671.

Williams, Q., and E.J. Garnero (1996) Seismic evidence of partial melt at the base of the mantle. Science, 273, 1528-1530.

Yagi, T., K. Okabe, N. Nishiyama, A. Kubo, and T. Kikegawa (2004) Complicated effects of aluminum on the compressibility of silicate perovskite. Phys. Earth Planet. Inter., 143-144, 81-91.

Zhao, D. (2001) Seismic structure and origin of hotspots and mantle plumes. Earth Planet. Sci. Lett., 192, 251-265.

Zhao, D. (2004) Global tomographic images of mantle plumes and subducting slabs: Insight into deep Earth dynamics. Phys. Earth Planet. Inter., 146, 3-34. 